\title{
LA POLÉMIQUE MÉDIATIQUE COMME LIEU DE CONFRONTATION POLITIQUE CONTEMPORAIN
}

\author{
Marc Lits ${ }^{1}$
}

Dans la tradition critique abondamment développée depuis les travaux fondateurs de Jürgen Habermas sur l'espace public, il est devenu commun de considérer que le débat public, tel qu'il se construisait dans la presse au XVIIIe siècle, a tendance à disparaître des colonnes des journaux, et plus encore dans les médias audiovisuels. Leurs propriétaires privilégieraient le profit, dans une logique capitaliste, au détriment de la discussion qui n'intéresserait guère les usagers. L'industrie culturelle et les entreprises de presse seraient donc vouées au divertissement, ce qui rejoint le point de vue de l'école de Francfort, tel qu'on peut le retrouver aussi dans les invectives de Pierre Bourdieu contre la télévision².

Pourtant, si cette tendance du glissement de la presse de sa fonction citoyenne, pour reprendre un terme aujourd'hui galvaudé, à

1 Marc Lits est professeur ordinaire à l'Université catholique de Louvain et directeur de l'Observatoire du récit médiatique.

2 Cf. J. HaBermas, L'Espace public. Archéologie de la publicité comme dimension constitutive de la sociêté bourgeoise, Paris, Payot, 1978 ; ID., "L'espace public", 30 ans après", Quaderni, n 18, automne 1992, pp. 161-191; P. BOURDIEU, Sur la télévision, Paris, Liber, 1996 ; É. GEORGE, "Du concept d'espace public à celui de relations publiques généralisées", Commposite, 1999, nº 1 (http://commposite.org). 
un rôle d'amuseur public, au service de la publicité, peut globalement être observée, elle ne peut être généralisée à toutes les époques et à tous les supports. Une fois de plus, un discours global, essentiellement fondé sur des a priori non vérifiés empiriquement, masque une part importante de la production médiatique réelle. Or, à regarder attentivement les lieux d'information, il apparaît qu'à certains moments, et dans certains espaces, le débat contradictoire reste très présent, y compris dans des affrontements de type polémique, souvent suscités par les médias eux-mêmes.

Bien sûr, l'évolution des chaînes de télévision, entraînée à la fois par les logiques de marchandisation et la multiplicité de l'offre, a comme conséquence, parmi d'autres, la disparition progressive des émissions à contenu politique, particulièrement les débats et autres lieux de confrontations idéologiques. Dans le même temps, la presse écrite d'opinion a tendance à céder du terrain au profit de quotidiens moins engagés (L'Humanité, La Croix, dans le cas de la France, résistent difficilement au déclin de la presse, comme Libération dans une moindre mesure), voire à quasiment disparaître là où les marchés sont plus restreints, comme en Belgique francophone ou en Suisse romande. Pourtant, les confrontations politiques continuent à nourrir les discussions autour de grands débats de société, particulièrement lors des échéances électorales. Certains enjeux démocratiques, comme la montée de l'extrême droite dans les pays occidentaux, ou le rôle de la religion dans des sociétés laïques, restent bien présents dans l'espace public. Ils doivent donc, d'une manière ou d'une autre, être mis en débat dans les médias classiques, dans la mesure où les sites web consacrés à des questions politiques, les forums et chats sur Internet restent encore des lieux d'expression minoritaires.

Il s'agit de voir d'abord où s'est déplacé le débat politique à la télévision, puisque cela reste le média dominant, comment il s'est ajusté à cette nouvelle organisation de l'espace public médiatique, en quoi ces transformations modifient à la fois la forme d'intervention et le contenu des argumentaires politiques. Mais il faudra aussi, dans un second temps, revenir à la presse écrite, parce qu'elle reste encore et toujours le lieu privilégié de la confrontation d'idées. Enfin, il est certain que cette évolution du débat politique médiatisé a des implications sur le système politique dans son ensemble, au moment où tous les partis et hommes politiques prennent de plus en plus en compte la gestion de leur communication politique. Dès lors, les enjeux éthiques 
liés au rôle de médias dans la vie publique sont indéniables, puisque ceux-ci organisent autrement le rapport des citoyens à la politique.

\section{Disparition progressive de la presse d'opinion}

Dans l'immédiat après-guerre, la Belgique et la France vont voir renaître une presse soucieuse de vivifier le débat démocratique, qui veut à la fois défendre des opinions très clairement marquées, fondées sur les valeurs d'un groupe politique ou social à l'engagement déclaré, mais aussi toucher l'opinion, voire l'influencer, sinon la construire. Certes, cette presse d'opinion va progressivement perdre son lectorat, puisque Combat, en France, disparait en 1974, alors que La Cité, en Belgique, arrive à survivre jusqu'en 1995, grâce à l'appui financier des syndicats chrétiens ${ }^{1}$.

Ceci infirmerait pour une part les affirmations pessimistes des disciples d'Habermas, telles qu'elles s'expriment, par exemple, dans les colonnes du Monde diplomatique, puisqu'aux moments cruciaux, le débat démocratique trouve, ou retrouve, sa place dans les médias. Mais ces moments seraient toujours limités, comme si les conditions de production et surtout les seuils de rentabilité l'emportaient toujours sur les motivations d'information et de formation démocratique. La fin du débat serait aussi liée à un désengagement généralisé par rapport à la chose politique, à la mort lente de la presse écrite, d'information générale comme d'opinion, à des changements d'attitude et de consommation du public, à une mauvaise adaptation d'un "produit" face à un lectorat en complète révolution. Toutes ces questions tournent autour de la place de l'engagement dans une société désenchantée et des rapports entre l'opinion et le marché.

Ce désenchantement du monde, le journaliste Jean-Claude Guillebaud le lie au reflux des grands systèmes idéologiques et à la prise de distance d'une génération d'intellectuels dont la dénonciation de la "pensée bourgeoise" était imprégnée d'une ritualisation magique qui empêchait tout regard critique. Il faudra toute la décennie 70 pour sortir "de trois grandes périodes manichéennes : fin de l'après-guerre, fin du "péché" colonial, fin de l'affrontement bipolaire entre l'Est et

1 Cf. F. Antolne (ed.), Coupures de presse. Disparition de La Cité et survie des médias d'opinion, Louvain-la-Neuve, Academia Bruylant, 1996. 
l'Ouest"'. Cette liquidation des oppositions manichéennes a entraîné la fin des "maîtres-penseurs" dénoncés par André Glucksmann, mais elle sonne aussi la disparition des conflits d'opinion. Quand la lutte des classes est reléguée dans les poubelles de l'histoire, meurent avec elle les lieux où se développaient les querelles de chapelles, à savoir essentiellement la presse imprimée qui vivait finalement de ces tensions antagonistes. La classe intellectuelle était toujours prompte à prendre la plume pour défendre une cause ou attaquer un ennemi objectif. Guillebaud, dans son essai, se réjouit de cette disparition des politiques partisanes au profit d'une réappropriation du quotidien par des sous-groupes de type associatif. Mais ceux-ci se constituent autour de projets très segmentarisés plutôt qu'en fonction d'une vision politique globale.

Face à cette concentration imposée par les lois du marché, et au moment où les sous-groupes se diversifient en se référant à des choix de vie (plutôt qu'à des idéologies) toujours plus éclatés, le paradoxe de la presse d'opinion atteint son sommet. Le journal ne présente plus une opinion (la sienne) au lecteur mais lui offre un panachage de l'ensemble des opinions susceptibles d'être imaginées par tous les lecteurs. Il est vrai que si l'on observe, par exemple, les rubriques d'opinion d'un journal comme Le Soir en Belgique, qui se déclare indépendant ou neutre, on y trouve surtout une multiplicité d'opinions clairement affichées, mais disparates, et réparties autant dans les caricatures que dans le courrier des lecteurs, les "cartes blanches", les éditoriaux ou la rubrique d'interviews. En France, Libération brasse également des positions parfois très antagonistes dans sa rubrique "Rebonds", sans chercher à les arbitrer. Autant de sensibilités très engagées qui se fondent sur une argumentation mêlant analyse politique et sensibilité personnelle, mais qui ne sont pas reliées à l'opinion du journal.

Quant à la télévision, l'insertion du discours politique au sein de ses émissions repose évidemment sur d'autres logiques, puisque les télévisions publiques doivent nécessairement laisser un espace aux différents partis, de la majorité et de l'opposition, et que les télévisions privées ne sont pas non plus immédiatement liées à une opinion politique représentative d'un parti (quoique la situation italienne actuelle oblige à nuancer fortement ce postulat que le marché serait

1 J.-Cl. GullebBaud, Les années orphelines. 1968-1978, Paris, Éd. du Seuil, 1978, p. 88. 
indépendant du politique). En outre, les changements de majorité politique, dans les systèmes électoraux qui favorisent l'alternance, entraînent aussi des repositionnements dans les rédactions des chaînes publiques, dont les responsables et parfois même les journalistes, sont pour partie nommés par les autorités politiques. Les commentaires, les prises de position connaîtront donc des variations au fil des différentes législatures. Enfin, là aussi, les tensions entre débat public et divertissement sont vives, davantage encore qu'en presse écrite.

\section{Un changement de format}

La prise de parole politique dans les médias a évolué au cours des cinquante ans d'histoire télévisuelle, à la fois parce que le support a beaucoup changé, mais aussi parce que la manière de faire de la politique s'est transformée dans une société dite de la post-modernité où le clivage entre les hommes politiques et les citoyens apparaît de plus en plus grand, suite à la dépréciation des grands "récits de légitimation". Dès lors, trois éléments changent en parallèle: le discours politique évolue et s'adapte aux nouveaux supports, le rapport du citoyen à la chose politique se transforme, puisque les modèles politiques globaux vont cesser d'attirer l'adhésion des citoyens davantage sensibles à des regroupements en sous-ensembles aux intérêts plus parcellaires, plus individualistes. Mais le traitement télévisuel de la parole politique se modifie aussi, parce que les conditions techniques permettent d'autres approches, et parce que le média tient compte des mutations sociales.

En Belgique, lorsque la majorité socialiste/sociale-chrétienne est remplacée en 1999 par un gouvernement "arc-en-ciel" présidé par un Premier ministre libéral, celui-ci annonce aussitôt qu'il veut privilégier une nouvelle culture politique axée sur le contact avec le citoyen et la transparence. Un certain nombre de journalistes, issus autant de la presse écrite que des radios et télévisions publiques ou privées, sont d'ailleurs engagés pour servir de porte-parole et travailler à cette communication politique renouvelée. Depuis ce moment, un discours très ambigu se met en place, typique des stratégies de communication importées du monde de l'entreprise. Une idée tente de s'imposer, un véritable mythe au sens où Barthes emploie le terme, qui voudrait qu'une plus grande communication des partis et des hommes politiques soit le garant d'un meilleur exercice de la citoyenneté, comme 
si plus de communication signifiait automatiquement une meilleure information du citoyen, et un meilleur échange entre ceux qui gèrent la cité et ceux qui y vivent. Alors que le terme "communication" est employé ici fort à propos, pour signifier que le monde politique gère désormais de manière professionnelle ses relations extérieures, comme le fait toute entreprise soucieuse de son image et de son chiffre d'affaires. Les hommes politiques ont appris à vendre leur image, à construire leurs discours publics, à définir des tactiques de campagne en fonction des attentes supposées du public. Ce n'est d'ailleurs pas un mal en soi, pour autant qu'on ne vende pas une stratégie de communication comme si c'était un progrès en termes d'information et de démocratie ${ }^{1}$.

Plus il y a d'agents de communication, dans les partis, aux côtés des ministres ou bourgmestres, plus il est important de former le citoyen à décoder le message politique qui passe sous de nouvelles apparences. La communication, en effet, peut privilégier la séduction plutôt que l'argumentation, choisissant ainsi la voie de la facilité, qui peut s'appeler proximité dans une vision positive, ou démagogie dans des usages moins nobles. Philippe Breton a bien rappelé combien ces procédés fusionnels doivent être utilisés avec prudence, insistant sur la nécessité de réapprendre les outils de l'argumentation afin d'envisager l'orateur comme responsable et garant de la liberté de l'auditoire $^{2}$. Cette condition permettrait de limiter l'accès à l'espace public à ceux qui respectent ce contrat. Le but, en effet, n'est pas de refuser d'emblée les évolutions stratégiques de la communication politique, mais de les appréhender avec le discernement critique dont devrait disposer tout citoyen formé au débat démocratique.

Si le marketing politique s'affine en Europe, et si le citoyen doit apprendre à interpréter ce nouveau type de message qui lui arrive, les chaînes de télévision ont elles aussi intégré ces évolutions. Elles tentent de se dégager de formules stéréotypées, pour entrer davantage dans la vie privée de l'homme politique, pour l'empêcher de pratiquer la langue de bois au profit du "parler vrai", pour le mettre davantage en prise directe avec le citoyen. L'homme politique lui-même va chercher à sortir du cadre rigide des émissions politiques pour apparaître dans des talk shows, des émissions de variété. Le rendez-vous

1 Cf. M. LiTs et S. Sepulchre, "Caquetages dans le poulailler gouvernemental", Médiatiques, $\mathrm{n}^{\circ} 17$, automne 1999, pp. 31-35.

2 Ph. Breton, La parole manipulée, Paris, La Découverte, 1997. 
politique du dimanche soir n'est plus à $19 \mathrm{~h}$ sur TF1, mais chez Michel Drucker. Cette tentative de rapprochement entre les politiques et les citoyens se manifeste aussi dans l'émission programmée par France 2 le dimanche midi et animée par Rachid Arhab. "J' ai rendez-vous avec vous", où il quitte le plateau et sort des studios pour se promener, caméra numérique à l'épaule, sur des marchés, à la rencontre des gens ordinaires. Ce n'est d'ailleurs pas un hasard si le premier numéro du 29 octobre 2000 portait sur le divorce entre les citoyens et la politique.

En ces temps où les taux d'abstention sont à la hausse, le réinvestissement dans la chose publique semble passer par des logiques de proximisation. Les affiches de campagne montrent le candidat entouré de sa femme et de ses enfants, les photographes de presse évitent les clichés guindés au profit des mises en situation (cf. la série du quotidien Le Soir pour les élections de 1999, qu'il reprend sous une autre forme en $2003^{1}$ ), les interviewers cherchent à faire apparaître l'homme qui se cache derrière le politicien. C'est la stratégie qu'emploient autant la télévision publique belge dans "Signé Dimanche" que la chaîne privée, quand elle charge Pascal Vrebos d'aller tous les dimanches midis à la rencontre des hommes politiques.

Ainsi, le marketing politique inspiré par les modèles de Jacques Pilhan $^{2}$, le défunt conseiller de François Mitterrand et de Jacques Chirac, est relayé par les politiques éditoriales des chaînes de télévision. Les deux camps tentent de se réconcilier avec le citoyen, même si c'est aussi pour retrouver des électeurs chez les uns, et des téléspectateurs chez les autres. Ce ne sont pas des objectifs contradictoires, pour autant qu'ils s'avouent comme tels. Le fait que la stratégie du parti belge Ecolo ne soit plus aussi rigide dans ses campagnes électorales, en acceptant avec modération une certaine personnalisation des candidats, n'apparaît pas comme un reniement de ses principes, mais comme un compromis entre des choix idéologiques et des stratégies électorales. On peut accepter que des idées passent aussi à travers des visages, que les engagements d'un parti se construisent avec des hommes et des femmes, qu'il ne s'agit pas de pures idéalités désincarnées. Mais le débat entre vie privée et vie publique, qui inspire des méta-discours télévisuels bimensuels chez Mireille Dumas, est assez

I Ph. Marion, "Politiciens clichés", Médiatiques, n 21, automne 2000, pp. 11-14.

2 J. Pilhan, "L'écriture médiatique", Le Débat, n 87, nov.-déc. 1995, p. 11. 
sensible pour qu'on connaisse aussi les limites de ces affichages ambigus. Pourtant, si la politique s'affiche comme un spectacle à la télévision, privilégiant la proximité au détriment du débat d'idées, la polémique ne disparaît pas pour autant de la place publique, du moins en période électorale. Mais elle se construit autrement, et surtout dans les colonnes des quotidiens, comme le montre l'analyse détaillée d'un débat politique récent, largement répercuté par la presse belge ${ }^{1}$, et qui nous servira ici d'exemple pour notre étude de cas.

\section{Le thème de l'intégration lance la campagne}

La disparition de la culture du débat et de la polémique, manifeste en télévision (puisque c'est un média fédérateur qui privilégie la culture du spectacle et qui s'adresse à un dénominateur le plus commun possible, à la différence du quotidien qui a encore des lectorats distincts) semblait aussi contaminer la presse écrite. Pourtant, les périodes électorales restent des moments particuliers où le goût de la confrontation, de la polémique revient en force, à la fois dans les partis politiques et dans les médias qui attisent parfois les querelles partisanes. Depuis que les spécialistes de science politique expliquent, pour partie, l'échec de Lionel Jospin aux élections présidentielles de 2002 par son manque d'affirmation d'une position tranchée, les conseils des experts en communication politique ont changé. Auparavant, il ne fallait rien faire pour effaroucher un électorat dispersé et volatil, mais plutôt tenir des propos centristes et rassembleurs. Aujourd'hui, cette position aseptisée montre ses limites et c'est le retour des clivages anciens entre gauche et droite, des positions marquées assénées avec force et conviction.

Le 8 janvier 2003, le Premier ministre belge Guy Verhofstadt annonce que les élections législatives auront lieu le dimanche 18 mai. Il met ainsi fin à une période de dégradation progressive du climat politique, dans la mesure où la fin de la législature entraînait une forme de surenchère entre les différents partis au pouvoir, chacun d'eux voulant s'attribuer les mérites exclusifs de mesures populaires (la diminution des impôts, par exemple) et imputant aux autres des

1 Le corpus reprend les deux quotidiens belges francophones de référence, La Libre Belgique et Le Soir, pour la période du lundi 14 octobre au samedi 19 octobre 2002, ainsi que les journaux télévisés du soir de la chaîne publique RTBF et de la principale chaîne privée RTL-TVI. 
décisions plus discutées (la suppression du grand prix de formule 1 à Francorchamps). Désormais, l'échéance est connue, et il ne faut plus essayer de faire endosser à l'un ou l'autre parti la responsabilité d'une chute du gouvernement avant le terme prévu. Mais cette annonce ouvre aussi la campagne électorale, même si celle-ci ne doit débuter officiellement que trois mois plus tard.

Ainsi, alors que le Mouvement Réformateur (MR) se présente comme un parti centriste et humaniste, où le Vice-Premier ministre Louis Michel incarne la défense ferme des droits de l'homme, son Président Daniel Ducarme avait déjà lancé un pavé dans la mare, le dimanche 13 octobre 2002. Invité sur le plateau de l'émission de débat de la chaîne privée RTL-TVI, Controverse, il y avait défendu fermement la note de réflexion et d'analyse politique sur l'intégration déposée peu auparavant par la Présidente du Parlement de la Communauté française, Françoise Schepmans (MR comme lui).

Cette note conclut à un échec de la politique d'intégration. Le débat en reste là, et est peu commenté dans les quotidiens du lundi, sinon dans le compte rendu que Le Soir donne des émissions de débats politiques du week-end, sous le titre explicite "La campagne servie sur deux plateaux", aussitôt précisé : "Positionnements en tous genres : c'est l'heure de la campagne électorale. En témoignaient hier Mise au point et L'invité. A la RTBF, Elio Di Rupo place son pôle des gauches à chaque réponse comme un uppercut. (...) Sur la chaîne concurrente, Daniel Ducarme, président du MR, a choisi, lui, de stresser les amis (...). Au passage, on apprend aussi que l'intégration des populations d'origine étrangère $a$ échoué, que les Belges ne sont plus bien chez eux, et que ce sera là un thème de campagne du MR."

Ce qui n'était qu'une déclaration parmi d'autres, aussi vite oubliée, va cependant connaître un retentissement considérable, au sein des partis et dans l'opinion, parce que la rédaction du Soir a saisi qu'il y avait là une rupture avec la convention tacitement acceptée par tous les partis francophones. Face à l'enjeu électoral qu'était devenu le thème de l'insécurité en France, aux risques de dérapages que cela entraînait par rapport à la thématique de l'immigration, et à la récupération possible par l'extrême droite, il était convenu que la prochaine campagne n'aborderait pas frontalement cette question, pour éviter de la caricaturer et de faire des immigrés un élément de marketing électoral. Le quotidien décide de revenir sur ce thème, et c'est donc bien un média qui le relance, en décidant de solliciter une interview plus approfondie du président Ducarme sur cette seule question. Cela 
fera la une de l'édition du mardi 15, sous le titre "Daniel Ducarme : "L'intégration est un échec"',

Le journal dévoile explicitement que c'est à son initiative que le débat est relancé, quand il écrit que "le président du Mouvement réformateur (MR) vient de rompre une lance. En télévision d'abord, furtivement. Dans nos colonnes ce jour, plus explicitement". Daniel Ducarme développe longuement son hypothèse qu'il y a "un problème par rapport à la politique d'immigration" et veut plaider, dit le journal, "pour que l'on ouvre le débat, entre formations démocratiques".

\section{Débat ou polémique?}

Une intervention catégorique, mais limitée à quelques phrases isolées dans un débat télévisé plus large, prend alors une tout autre ampleur. Partis politiques et mouvements associatifs se voient obligés de réagir, d'autant plus qu'ils vont être sollicités par l'ensemble de la presse, qui considère que le sujet est désormais à l'agenda. Durant une dizaine de jours, prises de position publiques, articles de journaux, séquences de journaux télévisés vont faire entrer la thématique de l'immigration dans la pré-campagne électorale, sur un ton qui relève davantage de la polémique que du débat contradictoire.

Dès le mardi soir, le journal télévisé de la RTBF fait écho à cette interview et aux réactions virulentes qu'elle suscite dans les autres partis. Les termes employés relèvent d'emblée du registre de la polémique, en portant plus sur la confrontation et les réactions personnelles que sur le fond du débat. Un des interviewés déclare par exemple: "On peut avoir des tendances de droite ou de gauche, mais il ne faut pas oublier avant tout d'être un démocrate et là j'ai l'impression que ce pauvre bonhomme perd un peu le nord". Joëlle Milquet, présidente du CDH, insiste : "C'est terriblement dangereux de sortir des choses aussi fausses comme cela maintenant car ça pourrait vraiment renforcer les thèses de l'extrême droite et en période électorale je trouve cela d'une démagogie extrême (...)". La journaliste conclut en disant que "Daniel Ducarme, dans son bureau, se sent diabolisé".

Sur RTL-TVI, le mercredi 16, le ton est aussi à la polémique et à l'attaque personnelle, quand le journaliste évoque la réaction de Philippe Moureaux : "Les propos tenus par le président du MR au 
sujet de la politique d'intégration dans l'émission Controverse ont fait sortir la fédération bruxelloise du Parti Socialiste de ses gonds. Son président, le bourgmestre de Molenbeek Philippe Moureaux, a accusé aujourd'hui Daniel Ducarme d'utiliser la voie de la rumeur pour diffuser des idées dangereuses (...). Chez les socialistes, l'affirmation du président du MR (...) sonne un peu comme une attaque frontale. (...). "Ce qui me frappe, c'est l'affirmation très forte proférée d'emblée par Daniel Ducarme, et puis après c'est le vide. Il n'y a aucun élément de démonstration, aucune solution. Cela s'appelle la rumeur : vous dites quelque chose, vous n'en dites pas plus et vous spéculez sur les effets que cela aura chez les gens", a expliqué Philippe Moureaux".

Cependant, si la première réaction est d'ordre polémique dans le lexique utilisé, dans les attaques ad hominem, dans la diabolisation des adversaires, le journal télévisé du lendemain essaye de contextualiser le débat, en donnant la parole à plusieurs élus du MR eux-mêmes issus de l'immigration et en proposant une séquence qui tente de définir ce qu' on entend par immigration. Et si le jeudi 17, le JT n'en parle plus, le lendemain, Louis Michel vient remettre de l'ordre dans ces échanges en tout sens, en rappelant la doctrine d'accueil du MR et que c'est lui qui contrôle encore le parti. "Je ne considère pas cela, dit-il, comme un incident majeur, je considère cela comme un incident de parcours, dont je ne pense pas que l'on puisse lui lancer une responsabilité quelconque là-dessus. Je pense honnêtement que sa conviction, c'était d'ouvrir un débat sur l'accès à la citoyenneté." Le journaliste annonce aussi que le Vice-Premier ministre "dénonce l'agressivité politique de certains pour stigmatiser le MR en qualifiant Louis Michel d'exception ou d'arbre qui cache la forêt". Ainsi la volonté de sortir de la polémique pour privilégier le débat est claire. Ce sera aussi la position de Daniel Ducarme quand il sera l'invité du débat politique du dimanche midi de la RTBF sur ce thème sensible. Peu à peu, ce qui avait surgi dans l'émission politique du dimanche de la chaîne privée trouvera sa fin (provisoire) dans l'émission parallèle du service public, le dimanche suivant. Avec une brève relance du débat, puisque la presse du lundi reviendra bien sûr sur les moments les plus sensibles de cette dernière confrontation télévisuelle. 


\section{Une polémique alimentée par les quotidiens}

Puisque Le Soir a allumé la mèche le mardi 15 , il contínue à alimenter le feu dès le lendemain, dans le registre de la polémique. Son titre de une est explicite : "Ducarme seul contre tous". Il évoque "un joli tollé dans la classe politique" et reprend dans son chapeau le terme d' "indécence" du communiqué commun des autres partis. La caricature de Kroll en p. 2 renforce cette logique d'affrontement puisqu'on y voit Ducarme enterré jusqu'à la tête et lapidé par les signataires de la réplique, déguisés en fondamentalistes musulmans. Le journal consacre encore une page et demie à ce sujet, partagée entre polémique politique et débat fondé à la fois sur des données chiffrées et des avis d'experts. Le grand titre en p. 4 est tout aussi représentatif de la tonalité : "Forte polémique autour de l'intégration". Les termes de "polémique", de "tremblement de terre", de "malaise", de "surenchère électorale", de "démagogie", de "rumeur pestilentielle" sont significatifs de l'état d'esprit des hommes politiques, des représentants de mouvements associatifs, voire des experts sollicités. Cependant, deux sociologues sont invités à témoigner de la réalité de l'immigration en Belgique, pour aider à construire le débat à l'aide d'arguments et de chiffres qui mettent à plat la question soulevée. Andrea Rea, par exemple, dissocie "ce discours électoraliste tonitruant" et "l'enjeu actuel, (...), savoir quelle société multiculturelle on veut défendre en Belgique".

Le jeudi, Le Soir continue dans la même logique : éléments de contextualisation (un topo sur la politique d'intégration et l'interview d'un immigré italien installé en Belgique depuis 45 ans) et analyse politique. Mais ici, les réactions virulentes sont entièrement occultées pour ne parler que des remous ("Les propos du président (...) continuent à faire des vagues") suscités par cette déclaration au sein du propre parti de Ducarme. Comme si, après l'orage, il fallait préparer l'accalmie. Le vendredi, un reportage réalisé rue des Coteaux à Bruxelles fait une plongée dans "l'intégration au quotidien" pour enfin donner la parole aux personnes directement mises cause dans le débat. Et le samedi, Le Soir semble vouloir mettre fin à la saga, en mettant à l'avant-plan Louis Michel qui "reprend la main et répond de Ducarme" (titre en une) et "contre-attaque" (titre de la p. 4). Si les termes de "polémique", de "semaine infernale" sont encore présents, 
le propos de Louis Michel se veut "centriste", comme la position du journal qui donne en parallèle une large place aux propos rassembleurs du Vice-Premier ministre et au portrait de Ducarme qui occupe la moitié de la p. 2, où il est qualifié simultanément de "malin, fragile et gaffeur".

Le Soir conclut d'ailleurs en refaisant l'historique de cette semaine, dans lequel il rappelle que c'est lui qui a relancé Ducarme le lundi. Il évoque surtout les réactions des différents partis quand ils voient que "la polémique s'emballe" et qu'ils "s'interrogent (...) à la lecture des médias" sur l'attitude à tenir. Il est intéressant de voir comment $L e$ Soir a travaillé en quatre temps : scoop avec l'interview exclusive de Ducarme, réactions violentes des autres partis francophones abondamment relayées, articles de contextualisation et de reportages, retour au calme proclamé par Louis Michel. Ainsi, le journal a pu à la fois faire surgir un sujet sensible politiquement, laisser la parole aux opposants, pour revenir sur le parti à l'origine du propos. Une histoire bouclée, où chacun s'est fait entendre (principalement, sinon quasi exclusivement, dans le monde politique) mais où la neutralité du quotidien se retrouve, puisque tous les points de vue ont été exprimés, dans un cycle classique de montée en puissance rapide suivie d'un progressif retour au calme.

La Libre Belgique entre dans le jeu avec un jour de retard, puisqu'elle n'a pas eu connaissance de l'interview de Ducarme avant sa sortie, et qu'elle intervient quand tous les politiques sont déjà entrés dans la polémique. Sa une du mercredi 16 titre donc "Daniel Ducarme provoque un ramdam politique", mais le sous-titre évoque aussi la réaction des autres partis qu'il "se met à dos". D'emblée, le journal situe la polémique dans le cadre de la pré-campagne, humant "le parfum d'un thème électoral". L'éditorial du rédacteur en chef condamne nettement ce qu'il qualifie d'"erreur d'analyse" sous le titre explicite "La faute politique et morale de Ducarme". Le jugement est sévère, envers celui qui est désigné comme un "apprenti sorcier, voire [un] démagogue". Cela n'empêche par le quotidien d'interviewer sur une demi-page le Président du MR, en face de l'interview du sociologue Marco Martinello. Et le reste de la classe politique a bien sûr aussi l'occasion de faire entendre son opposition à ces propos.

Mais dès le lendemain, le débat sur l'intégration est relayé au second plan, puisque l'essentiel de l'information est consacré à l'analyse politique de cette déclaration, avec une interview du président du FDF et une analyse de la situation au sein du MR. C'est cette option 
que privilégie le journal tout au long de la semaine. Informations sur les contacts entre le MR et le $\mathrm{CDH}$, débat entre la députée bruxellois Françoise Schepmans et son homologue socialiste Mahfoudh Romdhani, interviews des leaders libéraux Jacques Simonet et Didier Reynders. Pour finir, le samedi, avec le retour en force du "chef" Louis Michel. Il n'y a guère, ce même samedi, qu'une interview de la responsable du Centre pour l'égalité des chances, pour aborder le fond de la question de l'intégration des immigrés. Sinon, le dossier est essentiellement traité comme un fait politique, en donnant surtout la parole aux diverses personnalités du $\mathrm{MR}$, et en ne relayant guère les prises de position des opposants, qu'ils appartiennent aux autres partis ou au monde associatif et syndical. Quant aux personnes concernées sur le terrain, immigrés ou assistants sociaux, ils n'ont guère voix au chapitre. Mais il est vrai que la fin de semaine est aussi occupée par les remous au sein du syndicat socialiste bruxellois, et que plusieurs pages belges sont dès lors réservées à ce conflit et aux conférences de presse des différents protagonistes de la FGTB. Néanmoins, la position de La Libre Belgique apparaît quelque peu ambiguë. Elle condamne d'emblée les propos de D. Ducarme dans son éditorial, mais dans le même temps, elle n'accorde que peu de place aux réactions d'opposition, ou à la critique de ce point de vue, comme s'il s'agissait simplement d'un incident de campagne, qu'il fallait décrypter en fonction des enjeux pré-électoraux qui agitent le microcosme politique bruxellois. Sans vraiment prendre en compte l'impact de semblables propos sur l'opinion.

\section{Du bon usage de la polémique}

Quand les observateurs de la vie politique se désolent du désintérêt des citoyens pour les questions de société qui les concernent et regrettent de les voir se détourner de la politique, quand les analystes des médias observent le déclin de la presse et la disparition des médias d'opinion, on devrait se réjouir de retrouver des confrontations publiques autour de questions qui concernent directement l'opinion. Mais ce retour de la culture du débat ne peut se faire à n'importe quel prix. Si on analyse la manière dont s'est ici construit un débat emblématique, il faut remarquer que les questions éthiques et politiques qu'il soulève sont nombreuses. Il n'est pas certain que ces déclarations sur la manière dont la population immigrée est intégrée en 
Belgique soient entièrement guidées par le seul souci d'améliorer une politique sociale qui montre manifestement ses limites. La période pré-électorale ne peut faire négliger la part de calcul, voire de provocation, qui entre dans cette subite mise en avant d'un sujet ordinairement peu abordé sur la place publique. Mais cela relève de l'analyse politique.

Par contre, il faut nous interroger sur la manière dont les médias relayent, parfois suscitent, et ensuite exploitent ce type de thématique. Il est certain que cette question peut et doit être abordée dans des espaces de discussion qui permettent à chaque consommateur de médias, à chaque citoyen, de se construire une opinion par rapport aux grandes questions de société. Mais il faut aussi s'interroger sur les raisons qui poussent un quotidien à relancer, plutôt sur le mode polémique, une question aussi délicate, et sur la manière dont l'ensemble de la presse s'en empare. La seule question pertinente est liée à l'impact de cette polémique sur le public. Si celle-ci, au-delà des invectives et des règlements de compte politiques, permet de poser clairement la question de l'intégration, de faire l'état du dossier, de proposer des pistes de solution, alors les médias ont joué leur rôle social. Si les affrontements renforcent certains clichés négatifs, s'ils n'apportent aucun progrès dans l'amélioration d'une situation que chacun des intervenants considère comme insatisfaisante, il faut s'interroger sur la responsabilité sociale des uns et des autres.

Il ne s'agit pas de donner des leçons, mais de relancer, à l'occasion de cet épiphénomène de pré-campagne, une réflexion en profondeur sur le rôle conjoint des politiques et des médias dans la construction d'un espace public démocratique qui doit garantir une confrontation équilibrée, qui doit accepter la polémique, qui doit encourager la culture du débat, mais qui doit aussi favoriser les prises de position de chacun (et pas seulement des politiques entre eux) et faire émerger des solutions, des changements de société. Notre conclusion peut donc sembler paradoxale. En effet, les échanges politiques lors des campagnes électorales apparaissent de plus en plus aseptisés, puisque tous les partis visent à séduire des électeurs indécis en se plaçant au centre, à propos des grandes orientations de société. Et les campagnes relayent cette prudence, à travers des slogans insipides, une personnalisation souriante servant souvent de seule argumentation. Ce que l'on peut regretter, si l'on estime que le choix politique doit se fonder sur un arbitrage entre plusieurs options argumentées, renvoyant à des orientations précises témoignant de choix de société forts. Mais en 
même temps, nous semblons dénoncer les risques de polémiques malvenues sur des enjeux de société.

En fait, le débat doit exister, mais il ne peut être porteur de citoyenneté que s'il est posé en fonction d'enjeux politiques plutôt que politiciens. La frontière est ténue entre ces deux positions, mais elle peut être délimitée par l'implication des acteurs de la société civile dans ces débats. Si la confrontation ne se construit qu'avec des hommes de partis, au service de positionnements électoraux (du parti ou d'un individu en particulier), elle ne contribue guère à nourrir le débat public. Un des acquis de ces dernières années, dans le débat politique, est précisément la reconnaissance des acteurs de terrain, issus du monde associatif, de mouvements de base. Les médias n'ont pas encore tous intégré cette évolution, c'est pourtant en associant des intervenants divers, y compris en provenance de la société civile, qu'un nouvel espace public médiatique pourrait s'organiser. Mais non sans risques là aussi. Les réactions parfois virulentes à l'encontre des émissions de la RTBF animées par Paul Germain de 2001 à 2003, dans son "Lieu public", attestent de la difficulté à associer des hommes politiques et des individus dont la représentativité est parfois discutée et discutable. C'est bien à d'autres formes de confrontation médiatique qu'il faut donc réfléchir, en en mesurant les retombées publiques, les dérives démagogiques, les récupérations partisanes, les possibles manipulations. Entre le spectacle, l'émotionnel pur, les discours convenus des appareils politiques, le formalisme des émissions concédées aux groupements politiques et sociaux, l'aspect désuet des tribunes électorales, une place peut, -doit- être imaginée pour un débat médiatique représentatif des grands enjeux de société, captivant sans rendre le spectateur captif.

I Il s'agissait d'une émission mensuelle de débat autour d'un thème de société, animée en direct, et ouverte à toute personne qui souhaitait s'y exprimer, sans aucun filtre, avec une volonté délibérée de susciter des confrontations polémiques. Les incidents à répétition, entraînés par des intervenants tenant des propos extrêmes ou extrémistes, de type poujadiste, voire raciste, ont eu raison de l'émission. 